\title{
Functional role of a specific ganglioside in neuronal migration and neurite outgrowth
}

R. Mendez-Otero and M.F. Santiago
Instituto de Biofísica Carlos Chagas Filho,

Universidade Federal do Rio de Janeiro, Rio de Janeiro, RJ, Brasil
Correspondence

R. Mendez-Otero

Instituto de Biofísica, CCS, UFRJ

21941-590 Rio de Janeiro, RJ

Brasil

Fax: +55-21-2280-8193

E-mail: rmotero@biof.ufrj.br or

rmotero@abc.org.br

Presented at SIMEC 2002

(International Symposium

on Extracellular Matrix),

Angra dos Reis, RJ, Brazil,

October 7-10, 2002.

Research supported by PRONEX,

CNPq, FAPERJ, and by a grant

from the Ministry of Science and

Technology (MCT) to the Millennium

Institute for Tissue Bioengineering,

Brazil.

...................

Received February 5, 2003

Accepted May 27, 2003

\begin{abstract}
Cell migration occurs extensively during mammalian brain development and persists in a few regions in the adult brain. Defective migratory behavior of neurons is thought to be the underlying cause of several congenital disorders. Knowledge of the dynamics and molecular mechanisms of neuronal movement could expand our understanding of the normal development of the nervous system as well as help decipher the pathogenesis of neurological developmental disorders. In our studies we have identified and characterized a specific ganglioside (9-O-acetyl GD3) localized to the membrane of neurons and glial cells that is expressed in regions of cell migration and neurite outgrowth in the developing and adult rat nervous system. In the present article we review our findings that demonstrate the functional role of this molecule in neuronal motility.
\end{abstract}

Key words

- Neuronal migration

- Gangliosides

- Development

- 9-O-acetyl GD3

- Neurite outgrowth

\section{Introduction}

Directional movements occur extensively at all stages of morphogenesis of the nervous system. In addition to the interkinetic nuclear migration of the pseudostratified neuroepithelium, extensive cell migration occurs in the developing and adult nervous system and ensures that postmitotic immature neurons generated in the primary or secondary germinative zones acquire their proper positions in the mature brain. The first mode of migration - radial migration - involves movement of neuroblasts orthogonally to the pial surface, with most of the cells moving associated with a special glia, the radial glia. Guidance along radial glia is a common mechanism for the differentiation of projection neurons and the establishment of laminated structures, such as the cerebral cortex and the cerebellum (for reviews, see Refs. 1,2).

This migration process is a complex developmental program involving a series of steps including cell-cell recognition, cellcell adhesion, cell motility, and finally detachment from the glial fibers once neurons reach their destination (3). The other general migration mode - tangential migration - involves the displacement of neuronal progenitors or postmitotic cells parallel to the pial surface. This is a prominent feature in the migration of neuronal progenitors from the rhombic lip that are destined to brainstem nuclei or to the subpial aspect of the cerebellar cortex (4), in the migration of prospective luteinizing hormone-releasing hormone from the olfactory placode to the hypothalamus (5), of cortical interneurons from the gangli- 
onic eminences (6), and of prospective olfactory interneurons from the subventricular or subependymal zone to the core of the olfactory bulb (7). This mode of migration does not rely on a glial scaffold and several possibilities have been hypothesized to account for the migration of neuroblasts under such conditions. For example, it has been proposed that granular neuronal precursors migrate associated with axons to form the external granular layer of the cerebellum (8), and that some of the cells migrating tangentially from the ganglionic eminences to the cortex use the incoming corticofugal fibers as a guide (9). It has also been shown that in the rostral migratory stream neurons move rapidly along one another in unique chain formations independent of radial glia or axonal processes using their migrating neighbors to provide support for their movement (10).

These considerations raise the question of whether there are common molecular mechanisms underlying neuronal migration (radial or tangential) and other directional movements such as axonal pathfinding. In this respect, there is already evidence that some factors that control directional growth during tangential migration are similar to those that control the outgrowth of neuronal growth cones (for a review, see Ref. 11).

In our studies we have identified and characterized a specific glycolipid localized to the membrane of neurons and glial cells that is expressed in regions of cell migration and neurite outgrowth in the developing and adult nervous system. This molecule is recognized by a monoclonal antibody named Jones and was identified as the ganglioside 9-O-acetyl GD3 $(12,13)$. In several studies we have shown that the distribution of this ganglioside is temporally and spatially correlated with neuronal migration and neurite outgrowth in the central and peripheral nervous system and we thus hypothesize that this ganglioside participates in critical stages of neuronal migration and neurite outgrowth. In this review, we will summarize our stud- ies that provide evidence for the functional role of this glycolipid in neuronal motility.

\section{Gangliosides and neuronal motility}

Gangliosides are a large group of sialylated glycosphingolipids widely expressed in mammalian tissues and particularly abundant in the nervous system (14). Gangliosides possess a remarkable degree of structural diversity, and numerous enzymes are involved in their synthesis, recycling, and turnover. The biosynthesis of gangliosides proceeds in a stepwise manner starting from lactosyl ceramide and involves several glycosyltransferases. Some of the genes coding for some of the enzymes have been recently cloned but several questions are still under active investigation regarding how the levels of glycosyltransferases are regulated and determined (Figure 1A) (for a review, see Ref. 14). An important modification of gangliosides is the O-acetylation of sialic acid residues on C-9, C-7 or C-9 and C-7 observed on restricted cells and tissues but the enzymes responsible for these modifications (O-acetyl transferases) have been particularly difficult to clone. It has been suggested that $\mathrm{O}$-acetylation of gangliosides may serve as a cellular recognition signal and also as a tumor marker $(12,15)$. Sialidases are the key enzymes for ganglioside degradation and the plasma membrane-bound sialidase has been implicated not only in the general catabolism of gangliosides but also in the modulation of cellular function, such as proliferation and differentiation (16).

In the nervous system, the species and amounts of gangliosides undergo profound changes during development, suggesting that they may play fundamental roles in this process (17). The pattern of gangliosides changes from a relatively simple scheme, with GM3 and GD3 gangliosides predominating at earlier stages, to a more complex pattern with four gangliosides of the a- and b-series, GM1, GD1a, GD1b, and GT1b, that constitute the 
major species in the adult (for a review, see Ref. 18). Furthermore, the accumulation of gangliosides within the neurons in ganglioside storage diseases results in extensive neurite outgrowth (19) and exogenously administered gangliosides accelerate the regeneration of neurons in the central nervous system in vivo and stimulate cellular differentiation with concomitant neurite sprouting and extension in vitro (20). Recently, the genes involved in the biosynthesis of gangliosides have been cloned and genetically engineered mice or cells lacking most of the gangliosides have been produced (21). Surprisingly, the mice and the cells appeared to be largely normal in their neuronal development. However, more recent studies have revealed that mice lacking complex ganglio-

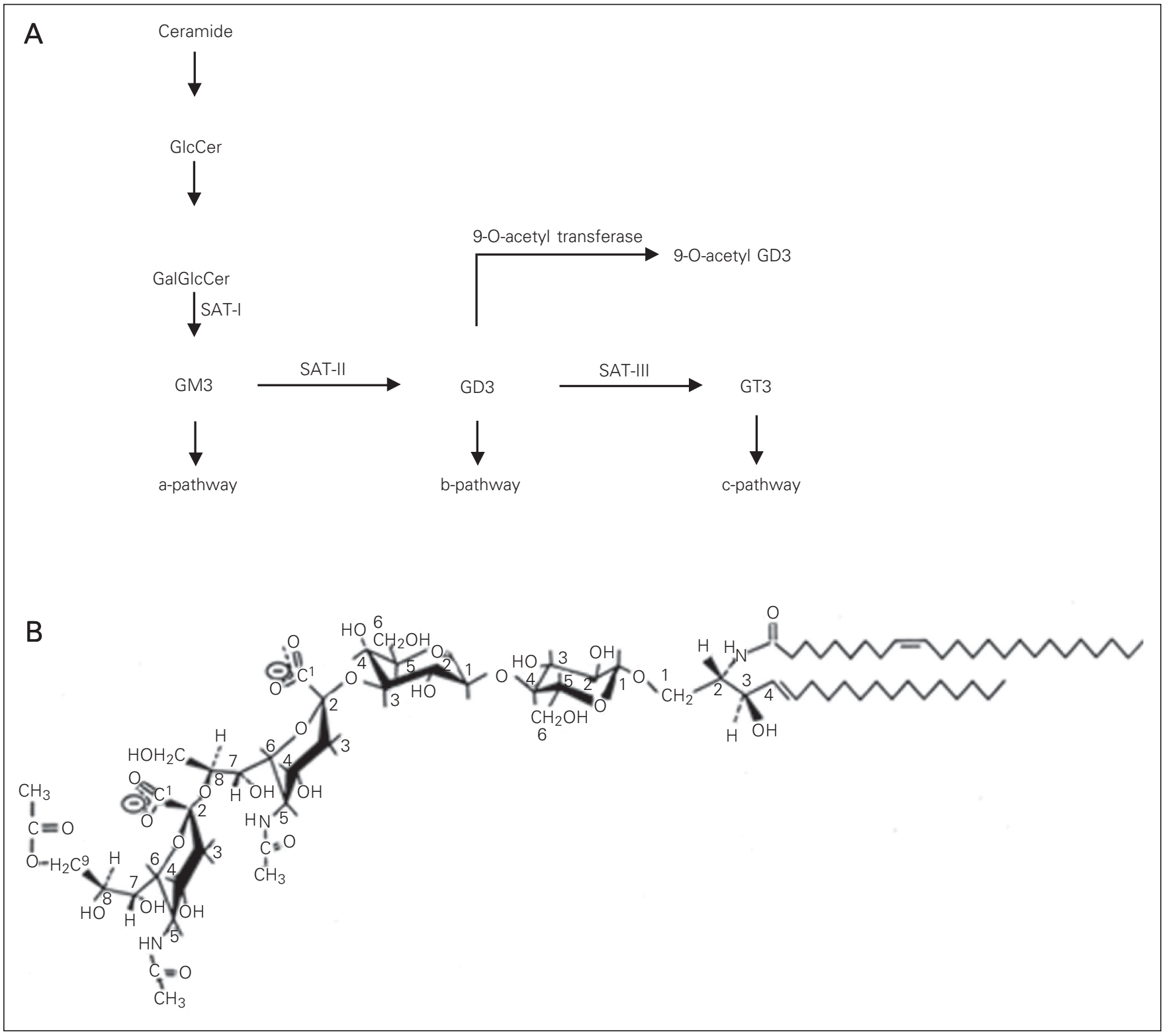

Figure 1. A, Proposed pathway for the biosynthesis of gangliosides. B, Schematic diagram showing the structure of 9-O-acetyl GD3. This ganglioside is formed by the addition of an acetyl group to the position 9 of the terminal sialic acid of GD3. Cer = ceramide; Gal = galactose; Glc $=$ glucose; SAT-I = GM3 synthase; Sat-II = GD3 synthase; SAT-III = GT3 synthase . 
Figure 2. Immunocytochemica distribution of 9-O-acetyl GD3 in the developing rat retina. Darkfield photomicrograph of an immunogold-stained section through the eye of an embryonic day 18 rat embryo. Observe the staining in the central part of the retina and the absence of labeling at the periphery. At this age the presumptive ganglion cells are formed in the central part of the retina and migrate to form the ganglion cell layer. Scale bar: $150 \mu \mathrm{m}$. sides exhibit axonal degeneration and myelination defects (22).

Experiments in several laboratories have shown that glycosphingolipids, gangliosides in particular, are important components of membrane rafts, where they can mediate important physiological functions such as cell adhesion and signal transduction events and consequently affect cellular phenotypes and functions (for a review, see Ref. 23). Recently, such microdomains have been termed "glycosynapses" in analogy to "immunological synapses" - the membrane assembly of immunocyte adhesion and signaling (24). Gangliosides are very abundant in glycosynapses and it has also been shown that anti-ganglioside antibodies can immunoprecipitate glycosylphosphatidylinositolanchored proteins (such as TAG-1), Src family kinases and caveolin $(25,26)$. In addition, several studies have shown the association of gangliosides with integrins and their ability to modulate integrin function (27).

Our studies have focused on a specific ganglioside recognized by the Jones monoclonal antibody (mAb) (12). In our initial studies we have shown that the pattern of staining with this antibody in the developing rat nervous system correlates temporally and spatially with neuronal migration and neurite outgrowth $(28,29)$. The biochemical char-

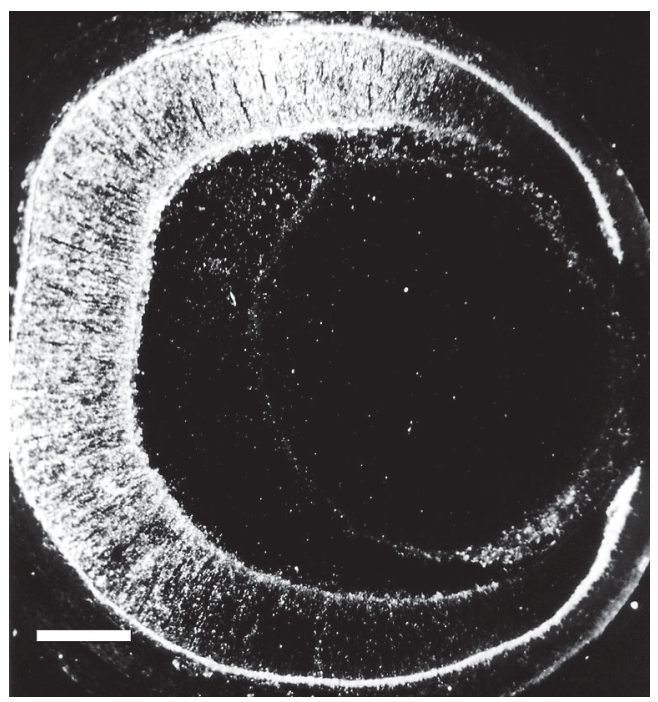

acterization of the antigen revealed that this $\mathrm{mAb}$ recognizes a ganglioside that migrates between GM1 and GM2 ganglioside standards. Further characterization using overlay assays on developed high-performance thin-layer chromatography plates indicates that the epitope recognized by the Jones $\mathrm{mAb}$ is expressed in several regions of the developing central and peripheral nervous system. In most regions examined the epitope resides in two bands (29). Further analysis has revealed that the most abundant and frequent band is 9-O-acetyl GD3, a modification of GD3 ganglioside in which an acetyl ester is formed at position 9 of the terminal sialic acid residue (Figure 1B) (13). The particular epitope recognized by the Jones $\mathrm{mAb}$ depends upon the acetyl group at position 9 of sialic acid and the immunoreactivity is abolished by mild base treatment since this treatment converts 9-O-acetyl GD3 to GD3 that is not recognized by the Jones $\mathrm{mAb}$ $(13,30,31)$. This antigen is abundant in the nervous system and in tumor cells derived from the neural crest, and is absent in most of the other tissues examined (29). However, the same epitope was described in the immune system and was designated CD60b (32). The 9-O-acetyl GD3 is present in the retinas of all mammalian species studied so far but is not present in extracts of frog retinas (33). At least two other mAbs have been described (mAb D1.1 and RB13-2) that recognize the same epitope in nervous tissue $(34,35)$. The immunoreactivity of the slower migrating band is also abolished by mild base treatment and it has been suggested that this band might correspond to 9-O-acetyl GQ1c (36).

In the developing nervous system, we have shown that the expression pattern of this specific Jones $\mathrm{mAb}$ reactive ganglioside correlates with times of cell migration in the retina (Figure 2), superior colliculus, cerebellum, and telencephalon and in regions undergoing neurite extension, such as the developing optic tract, the white matter of the cerebellum, the dorsal roots (Figure 3), 
the trigeminal system, and olfactory nerve $(12,28,29,33,37-39)$. This has led us to suggest that the function of 9-O-acetyl GD3 is to modulate motility either directly or by modifying the efficacy of some other component of the system $(39,40)$. The association of 9O-acetylated gangliosides with cell migration has been well characterized in cancer research, where it was found that tumors arising from neural crest-derived tissues express high levels of 9-O-acetyl GD3. These gangliosides are concentrated in adhesion plaques and are involved in cell adhesion and migration in tumor cells $(14,15)$. In addition, disialogangliosides co-immunoprecipitate with $\alpha \mathrm{VB3}$ integrin and GM1 coimmunoprecipitates with the epidermal growth factor receptor (for a review, see Ref. 24). Furthermore, it was also shown that cleavage of 9-O-acetyl groups by transgenic expression of influenza $\mathrm{C}$ virus hemagglutinin caused abnormalities in the development of the retinal layers in the mouse, suggesting a failure in cell migration concomitant with the absence of 9-O-acetyl GD3 (41). Recently, it was shown that down-regulation of 9-O-acetyl GD3 by stable transfection of Oacetylesterase cDNA and antisense vector against the GD3-synthase gene results in cell differentiation in melanoma cell lines (42). Based on our observations and the reports concerning other systems, we raised the hypothesis that 9-O-acetyl GD3 may play an important role in neuronal motility in the developing and adult nervous system. In the following sections we will describe the evidence showing a functional role for this ganglioside in neuronal migration and neurite outgrowth.

\section{Gangliosides in glial-guided radial migration}

In most cases, neuronal radial migration occurs on the processes of radial glial cells and has been studied extensively in the cortex and cerebellum although similar arrange- ments for migrating neurons and specialized glia have been described in a number of other systems including the retina and the hippocampus (for a review, see Ref. 1). Recent studies have begun to provide a framework for the molecular mechanisms underlying this mode of migration (for a review, see Ref. 43).

In the postnatal cerebellum, the postmitotic neurons in the external granular cell layer also migrate radially but in this case away from the pia towards the internal granular cell layer. This migration is also associated with radial glia and this system has been extensively used as a model for studies of glial-guided migration (for a review, see Ref. 1). We have investigated the possible role of 9-O-acetyl GD3 in the glial-guided neuronal migration using the developing rat cerebellum as a model. In previous studies we had shown that the expression of this ganglioside is developmentally regulated in the developing cerebellum (28). In E14-18 fetuses, immunocytochemistry labeling with Jones $\mathrm{mAb}$ is present, extending from the ventricular to the pial surface of the cerebellar anlage. Most of the immunoreactivity is distributed in a radially oriented pattern that corresponds to the radial migration of Purkinje cell precursors migrating from the ventricular zone towards the pial surface. Immunoreactivity, however, is present in the rhombic lip and in the developing external granular cell layer corresponding to the subpial tangential migration of these cells. During the postnatal period in the rat, the cells in the external granular cell layer proliferate and migrate

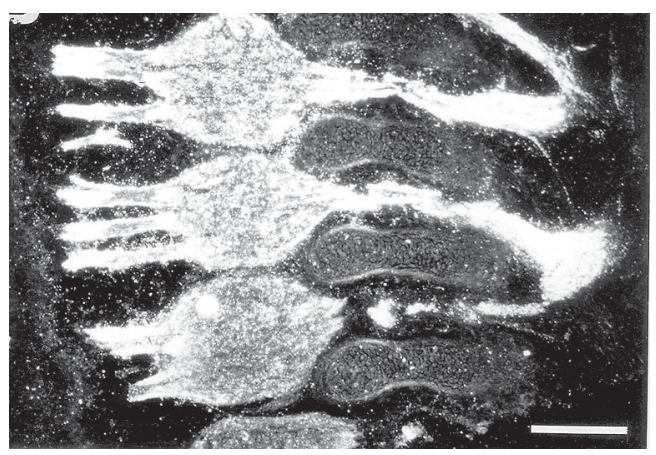

Figure 3. Pattern of 9-O-acetyl GD3 expression in the developing dorsal root ganglia. Darkfield photomicrograph of a parasagittal section through the cervical region of an embryonic day 18 rat. Observe the intense immunoreactivity in the ganglia and also in the central and peripheral processes. Scale bar: $50 \mu \mathrm{m}$. 
radially away from the pia to form the internal granular cell layer. At this stage, immunocytochemistry reveals a radially oriented pattern of Jones binding extending from the external granular cell layer towards the presumptive granular cell layer (Figure 4). Electron microscopic immunocytochemistry revealed that around the peak of cerebellar neuronal migration, 9-O-acetyl GD3 was localized at the contact sites between migrating granule cells and radial glia in the external granular cell layer and prospective molecular layer (40). We have also observed that both cultured neurons from the rat cerebellum when in contact with glial cells that support cell migration (radial glial cells) and glial cells themselves express 9-O-acetyl GD3 (44). In contrast, glial cells with a stellate morphology, even when in contact with granule neurons, do not express this antigen (44). To test the functional role of 9-O-acetyl GD3 in neuronal migration we have used cerebellar explants and cerebellar slices. We have shown that the Jones mAb blocks the migration of neurons in a dose-dependent manner, suggesting that 9-O-acetyl GD3 is involved in the radial migration of presumptive granule cells in the developing cerebellum (40). These results further support our view that this molecule is involved in cell migration.

The immunoreactivity for this ganglioside is also present in the embryonic telencephalon, showing a radial organization correlated with radial migration in this region (28). Blockage of the ganglioside with Jones antibody arrests neuronal migration on tissue slices of embryonic telencephalon (45).

Figure 4. Correlation of 9-Oacetyl GD3 expression with radial cell migration. Fluorescence photomicrograph of a parasagittal section through the cerebellum of a newborn rat. Rostral is to the right. Observe the staining of 9-O-acetyl GD3 throughout the presumptive cerebellar cortex in the most anterior folia and the absence of labeling in the posterior folia. Scale bar: $100 \mu \mathrm{m}$.

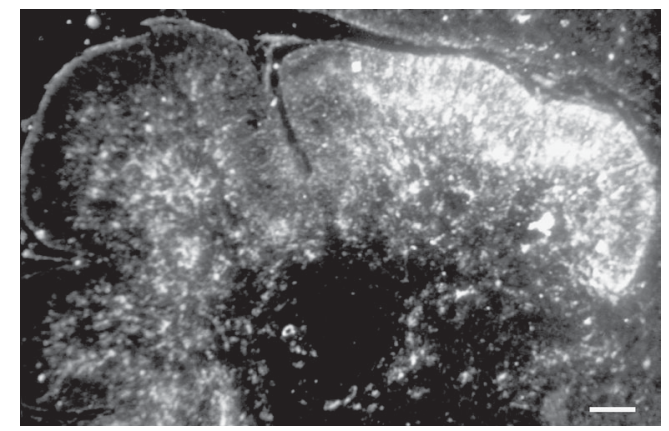

\section{Gangliosides in tangential migration}

Glial-guided neuronal migration has been relatively well studied, whereas much less is known about tangential migration (for a review, see Ref. 11). Tangentially migrating neurons are found in the cortex, the cerebellum and the rhombencephalon and they migrate perpendicularly to the glial scaffolding $(6,46,47)$. Some of the molecular cues that guide tangential migration have been recently identified and the picture that is emerging is that the same families of signals involved in the directional guidance of developing growth cones may also guide the translocation of cell bodies during tangential migration (9).

The migration of interneurons from the anterior subventricular zone to the olfactory bulb provides a useful system to study the cellular and molecular mechanisms that regulate tangential neural migration. These neuronal precursors migrate through a distinct pathway within the subventricular zone called the rostral migratory stream, and this migration occurs even in the adult. Molecular studies have shown that, in the particular case of the rostral migratory stream, the mechanism of migration involves the sialylated form of the neural cell adhesion molecule (NCAM) and is guided, in part, by negative chemotropism (48).

In view of the abundant evidence for a role of 9-O-acetyl GD3 in directional movements of neuronal soma or processes we have performed an immunohistochemical analysis of their expression along the route of tangential migrations of some olfactory bulb prospective interneurons in the developing and adult rats. We have found that this ganglioside is highly expressed in the lateral ventricle subventricular zone and along the route of tangential migration (rostral migratory stream) into the olfactory bulb during development (Figure 5). In the adult, we have found staining around the lateral ventricle and in chains of cells in the rostral migratory stream region (49). In a few sec- 
tions, individual stained cells are observed, with a morphology similar to that of migrating cells. Despite the scarcity of staining, this is one of the few regions where expression of 9-O-acetyl GD3 is found in the adult nervous system. Interestingly, this region has been described as one of the regions in which neurogenesis persists in the adult brain and from which stem cells can be isolated in the adult brain. It would be interesting to determine whether stem cells isolated from adult brains also express 9-O-acetyl GD3.

To investigate the functional role of 9-Oacetyl gangliosides in tangential migration we have used explants of the subventricular zone region as a model. We have found that migrating chains similar to the ones formed in vivo are also seen in this in vitro system. The migrating chains from the subventricular zone explant express 9-O-acetyl GD3 which is distributed in a punctiform manner in individual cells and treatment of the cultures with the antibody against 9-O-acetyl GD3 arrested neuronal migration in these cultures. These data suggest that 9-O-acetyl GD3 participates in neuronophilic as well as gliophilic migration $(50,51)$.

\section{Gangliosides and neurite outgrowth}

During development, neuronal growth cones interact with physical and chemical cues in their environment and these interactions guide the growth cones along specific pathways to their appropriate targets (for a review, see Ref. 52). Many factors such as soluble or substrate-bound growth factors and components of the extracellular matrix provide favorable environments that allow or promote motility of growth cones and neurite elongation (53). Repulsive and inhibitory factors have also been described and may participate in guidance of growth cones (for a review, see Ref. 52). Gangliosides, in particular disialogangliosides, have been implicated in adhesion systems and in cell to substrate adhesion of both melanoma and neuroblastoma cells (15). Indeed, a number of separate lines of evidence support the hypothesis that these molecules modulate the activity of the integrin family of cell surface adhesion molecules (for a review, see Ref. 23) and selectively induce the synthesis of MAP2 (high molecular weight microtubule-associated protein 2) (54). Furthermore, numerous studies employing a variety of models have reported that ganglioside treatment will increase the rates of neuritogenesis both in vitro and in vivo, and axonal regeneration and sprouting following brain trauma to the central or peripheral nervous system, suggesting a role for gangliosides in neurite outgrowth.

In our studies of the expression of 9-Oacetyl GD3 in the developing nervous system we have found that, in addition to the pattern of staining coinciding with neuronal
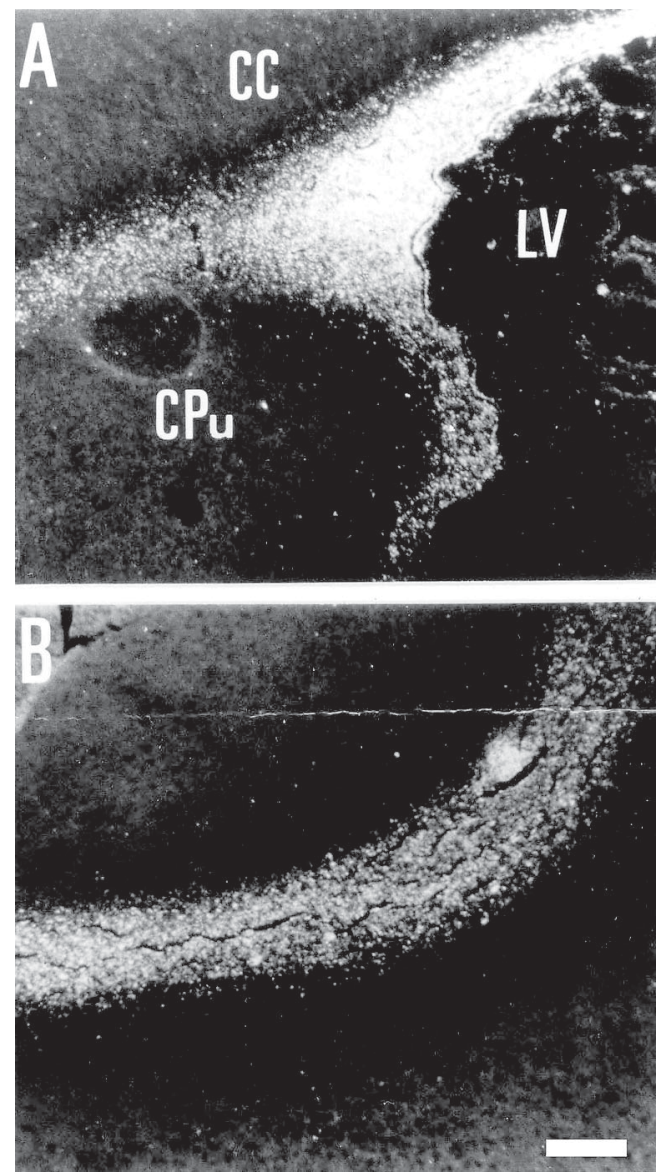

Figure 5. Expression of 9-Oacetyl GD3 is correlated with tangential cell migration. A, Photomicrographs of a parasagittal section through the telencephaIon of a postnatal day 7 rat stained with the antibody against 9-O-acetyl GD3. Note the intense staining around the lateral ventricle (LV) extending towards the olfactory bulb (to the left of the figure) and around the caudatum-putamen (CPu). CC, corpus callosum. $B$, The rostral migratory stream shows a high expression of 9-O-acetyl GD3. Scale bar: $100 \mu \mathrm{m}$. 
migration, these gangliosides were also observed along pathways of axon extensions such as the optic tract, the olfactory nerve and the central and peripheral processes of dorsal root ganglia. This pathway staining is only observed while large numbers of growth cones are present at these positions and the staining disappears from most of the pathways studied as soon as the axons reach their target regions $(28,37)$. One interesting exception to this generalization, however, is the olfactory system (37). In this system we have shown that the expression of 9-O-acetyl GD3 begins as early as embryonic day 13, when the olfactory epithelium and the migratory mass were intensely stained. At embryonic day 19, the immunoreactivity had disappeared from the olfactory epithelium but remained in a few fascicles and some glomeruli of the olfactory bulb in the newborn and adult. We conclude that the expression of 9-O-acetyl GD3 by olfactory axons and/or migrating cells may facilitate axonal outgrowth during development and might be involved in the formation of new glomeruli in the mature olfactory bulb (37).

We have recently investigated whether 9-O-acetyl gangliosides are also re-expressed during the regeneration of sciatic nerves that were submitted to crushing. We have found an up-regulation of 9-O-acetyl GD3 expression starting five days after crushing, reaching a maximum at day 7 , and decreasing afterwards to reach the level of the control animals at day 10 (Mendez-Otero R, Silva AO, Resende VTR and Hedin-Pereira C, unpublished data). These results suggested to us that 9-O-acetyl GD3 may play a functional role in neurite outgrowth during development and regeneration.

To test the functional role of 9-O-acetyl GD3 in neurite outgrowth, we used explants of dorsal root ganglia. In previous studies we have shown that embryonic dorsal root ganglion neurons and their processes either in vivo (28), in explant cultures or as dissociated cells present heavy immunoreactivity to the antibody against the ganglioside. We then plated dorsal root ganglion explants from embryonic day 16 onto laminin, collagen or fibronectin. Neurites grew out of the explants and expressed high amounts of 9O-acetyl GD3 in all substrates. We then investigated the functional role of this ganglioside on neurite extension using embryonic dorsal root ganglion explants grown on laminin substrates as a model. In these experiments, the behavior of individual growth cones was recorded using a time-lapse videoenhanced imaging system before and after the addition of antibodies that recognize specific gangliosides known to be expressed on these growth cones. Using this system, it was possible to demonstrate that the advance of growth cones on laminin was halted in the presence of Jones $\mathrm{mAb}$. The onset of the effect was rapid and signaled by an immediate cessation of elongation, a loss of lamellipodia and a retrieval of axoplasm (55). Blockade of 9-O-acetyl GD3 also induces the appearance of lateral spikes along the neurites in addition to the effect on the growth cone. We have shown that microfilaments and microtubular rearrangements accompany these structural modifications (56). Our results suggest that 9-O-acetyl GD3 may play an important role in the extension of growth cones and consequently influence navigation and pathway finding during development and regeneration.

\section{Conclusion and future directions}

The mechanisms by which ganglioside expression leads to neuronal migration and neurite outgrowth are still far from being completely identified. However, based on the data reviewed here and also on several findings from different laboratories, it is possible to suggest several mechanisms to explain the role of 9-O-acetyl GD3 in these phenomena. It is likely that specific gangliosides could interact with broadly distributed protein-based adhesion systems and favored 
subsets of moving cells and/or processes that express these molecules (Figure 6). Examples of such mechanism are the described interactions of gangliosides with integrin receptors $(57,58)$ and with TAG-1 (an adhesion molecule) (25). Another possibility is that gangliosides may serve to modulate the availability of divalent cations to a number of different calcium- or magnesium-dependent adhesion systems and consequently regulate ligand binding (57). Alternatively, it has been suggested that gangliosides may regulate the lateral diffusion and assembly of signaling complexes in membrane microdomains and therefore interfere with the functional role of these proteins, as proposed for integrin in focal adhesion points and TAG-1 (59). Another possible model is based on the homophilic interaction between gangliosides located on different cell membranes. A similar model has been proposed to explain the involvement of NCAM in regulating neural cell adhesion and axon growth. Finally, it has also been shown in several systems that selectins and/or galectins can function as receptors for specific gangliosides (60) and this interaction could trigger a cascade of reactions in both cells.

The ganglioside 9-O-acetyl GD3 could provide a new cell signaling mechanism in glial-guided neuronal migration in the developing nervous system. Moreover, it is important to note that most neurons migrate by extension of a leading, neurite-like process and that 9-O-acetyl GD3 has been impli-

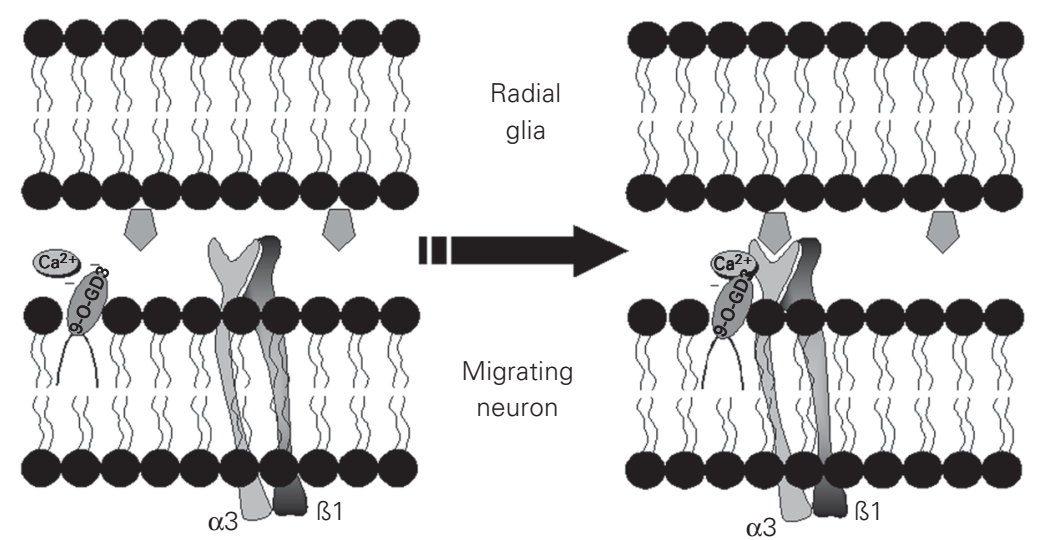

Figure 6. Schematic diagram suggesting possible mechanisms for 9-0-acetyl GD3 action on neuronal motility. The ganglioside may modulate the integrin receptor through a $\mathrm{Ca}^{2+}$ dependent mechanism and/or by a direct interaction between the ceramide moiety of the ganglioside and the integrin transmembrane helix. In a first stage, the integrin receptor is inactive and there is no recognition of extracellular matrix proteins. Later, gangliosides in the adjacent membrane become laterally packed around the integrin receptor (represented in the scheme by only one ganglioside), providing an optimal $\mathrm{Ca}^{2+}$-enriched microenvironment for the activation and recognition of extracellular matrix proteins by this receptor. In addition, the gangliosides can interact directly with the integrin receptor through a conserved lysine located inside a 23-amino acid sequence at the carboxy-terminus of the integrin transmembrane helix. 9-O-GD3 = 9-O-acetyl GD3.

cated in both neuronal migration and neurite outgrowth. The potential roles for this ganglioside in identical mechanisms for neuronal migration and neurite outgrowth suggest an emerging framework in which glycolipids are involved in cell movement in general.

\section{Acknowledgments}

The technical assistance of Felipe Marins is gratefully acknowledged.

\section{References}

1. Hatten ME (1999). Central nervous system neuronal migration. Annual Review of Neuroscience, 22: 511-539.

2. Parnavelas JG (2000). The origin and migration of cortical neurons: new vistas. Trends in Neurosciences, 23: 126-131.

3. Nadarajah B, Brunstrom JE, Grutzendler J, Wong ROL \& Pearlman AL (2001). Two modes of radial migration in early development of the cerebral cortex. Nature Neuroscience, 4: 143-150.

4. de Diego I, Kyriakopoulou K, Karagogeos D \& Wassef M (2002). Multiple influences on the migration of precerebellar neurons in the caudal medulla. Development, 129: 297-306

5. Norgren Jr RB \& Lehman MN (1991). Neurons that migrate from the olfactory epithelium in the chick express luteinizing hormone-releasing hormone. Endocrinology, 128: 1676-1678.

6. Tamamaki N, Fujimori KE \& Takauji R (1997). Origin and route of tangentially migrating neurons in the developing neocortical intermediate zone. Journal of Neuroscience, 17: 8313-8323.

7. Lois C, Garcia-Verdugo J-M \& Alvarez-Buylla A (1996). Chain migration of neuronal precursors. Science, 271: 978-981. 
8. Hynes RO, Patel R \& Miller RH (1986). Migration of neuroblasts along preexisting axonal tracts during prenatal cerebellar development. Journal of Neuroscience, 6: 867-876.

9. Denaxa M, Chan C-H, Schachner M, Parnavelas JG \& Karagogeos D (2001). The adhesion molecule TAG-1 mediates the migration of cortical interneurons from the ganglionic eminence along the corticofugal fiber system. Development, 128: 4635-4644.

10. Wichterle H, Garcia-Verdugo J \& Alvarez-Buylla A (1997). Direct evidence for homotypic, glia-independent neuronal migration. Neuron, 18: 779-791.

11. Corbin JG, Nery S \& Fishell G (2001). Telencephalic cells take a tangent: non-radial migration in the mammalian forebrain. Nature Neuroscience, 4: 1177-1182.

12. Constantine-Paton M, Blum AS, Mendez-Otero R \& Barnstable C (1986). A cell surface molecule distributed in a dorso-ventral gradient in the perinatal rat retina. Nature, 324: 459-462.

13. Blum AS \& Barnstable CJ (1987). O-acetylation of a cell-surface carbohydrate creates discrete molecular patterns during neuronal development. Proceedings of the National Academy of Sciences, USA, 84: 8716-8720.

14. Lloyd KO \& Furukawa K (1998). Biosynthesis and functions of gangliosides: Recent advances. Glycoconjugate Journal, 7: 627636 .

15. Cheresh DA, Varki AP, Varki NM, Stallcup WB, Levine J \& Reisfeld RA (1984). A monoclonal antibody recognizes an O-acetylated sialic acid in a human melanoma-associated ganglioside. Journal of Biological Chemistry, 259: 7453-7459.

16. Reitzenstein C, Kopitz J, Schuhmann V \& Cantz M (2001). Differential functional relevance of a plasma membrane ganglioside sialidase in cholinergic and adrenergic neuroblastoma cell lines. European Journal of Biochemistry, 268: 326-333.

17. Rosner H (1994). Gangliosides and brain development. In: Nicolini M \& Zatta PF (Editors), Glycobiology and the Brain. Pergamon Press, New York, 19-39.

18. Yu RK (1994). Developmental regulation of ganglioside metabolism. Progress in Brain Research, 10: 3-44.

19. Purpura DP (1978). Ectopic dendritic growth in mature pyramidal neurons in human ganglioside storage disease. Nature, 276: 520521.

20. Roisen F, Matta SG \& Rapport MM (1986). The role of gangliosides in neurotrophic interaction in vitro. In: Tettamati G, Ledeen RW, Sadhoff K, Nagai Y \& Toffano G (Editors), Gangliosides and Neuronal Plasticity. Liviana Press, Padova, Italy, 281-293.

21. Takamiya H, Yamamoto A, Furukawa K et al. (1996). Mice with disrupted GM2/GD2 synthase gene lack complex gangliosides but exhibit only subtle defects in their nervous system. Proceedings of the National Academy of Sciences, USA, 93: 10662-10667.

22. Chiavegatto S, Sun J, Nelson RJ \& Schnaar RL (2000). A functional role for complex gangliosides: motor deficits in GM2/GD2 synthase knockout mice. Experimental Neurology, 166: 227-234.

23. Pande $G$ (2000). The role of membrane lipids in regulation of integrin function. Current Opinion in Cell Biology, 12: 569-574.

24. Hakomori SI (2002). The glycosynapse. Proceedings of the National Academy of Sciences, USA, 99: 225-232.

25. Kasahara K, Watanabe K, Takeuchi K, Kaneko H, Oohira A, Yamamoto T \& Sanai $Y$ (2000). Involvement of gangliosides in glycosylphosphatidylinositol-anchored neuronal cell adhesion molecule TAG1 signaling in lipid rafts. Journal of Biological Chemistry, 275: 34701 34709.

26. Katagiri YU, Mori T, Nakajima H, Katagiri C, Taguchi T, Takeda T, Kiyokawa N \& Fujimoto J (1999). Activation of Src family kinase Yes induced by Shiga toxin binding to globotriaosyl ceramide (Gb3) CD77) in low density, detergent-insoluble microdomains. Journal of Biological Chemistry, 274: 35278-35282.

27. Yates AJ \& Rampersaud A (1998). Sphingolipids as receptor modulators: an overview. Annals of the New York Academy of Sciences, 845: 7-71.

28. Mendez-Otero R, Schlosshauer B, Barnstable CJ \& ConstantinePaton M (1988). A developmentally regulated antigen associated with neural cell and process migration. Journal of Neuroscience, 8 : 564-579.

29. Schlosshauer B, Blum AS, Mendez-Otero R, Barnstable CJ \& Constantine-Paton M (1988). Developmental regulation of ganglioside antigens recognized by the JONES antibody. Journal of Neuroscience, 8: 580-592.

30. Bonafede DM, Macala LJ, Constantine-Paton M \& Yu RK (1989). Isolation and characterization of ganglioside 9-O-acetyl-GD3 from bovine buttermilk. Lipids, 24: 680-684.

31. Ritter G, Boosfeld E, Markstein E, Yu RK, Ren SL, Stallcup WB, Oettgen HF, Old LJ \& Livingston PO (1990). Biochemical and serological characteristics of natural 9-O-acetyl GD3 from human melanoma and bovine buttermilk and chemically O-acetylated GD3. Cancer Research, 50: 1403-1410.

32. Mason D, Andre P, Bensussan A et al. (2001). CD antigens 2001 : Aims and results of HLDA workshops. Stem Cells, 19: 556-562.

33. Mendez-Otero R, Schlosshauer B \& Constantine-Paton M (1992). Role of acetylated gangliosides on neuronal migration and axonal outgrowth. In: Lent R (Editor), The Visual System from Genesis to Maturity. Birkhauser, Boston, MA, 49-62

34. Levine JM, Beasly L \& Stallcup WB (1984). The D1.1 antigen: a cell surface marker for germinal cells of the central nervous system. Journal of Neuroscience, 4: 820-831.

35. Reinhardt-Maelicke S, Cleeves V, Kindler-Rohrborn A \& Rajewsky MF (1990). Differential recognition of a set of O-acetylated gangliosides by monoclonal antibodies RB13-2, D1.1, and Jones during rat brain development. Developmental Brain Research, 51: 279-282.

36. Multani P, Bonafede DM, Yu RK \& Constantine-Paton M (1988). Biochemical characterization of Jones immunoreactive gangliosides in rat. Society for Neuroscience Abstracts, 14: 1016.

37. Mendez-Otero R \& Ramon-Cueto A (1994). Expression of 9-O-acetylated gangliosides during development of the rat olfactory system. NeuroReport, 5: 1755-1759.

38. Mello LEAM \& Mendez-Otero R (1996). Expression of 9-O-acetylated gangliosides in the rat hippocampus. Neuroscience Letters, 213: $17-20$

39. Mendez-Otero R \& Santiago MF (2001). Functional role of a glycolipid in directional movements of neurons. Anais da Academia Brasileira de Ciências, 73: 221-229.

40. Santiago MF, Berredo-Pinho M, Costa MR, Gandra M, Cavalcante LA \& Mendez-Otero $R$ (2001). Expression and function of ganglioside 9-O-acetyl GD3 in postmitotic granule cell development. Molecular and Cellular Neurosciences, 17: 488-499.

41. Varki A, Hooshmand F, Diaz S, Varki NM \& Hedrick SM (1991). Developmental abnormalities in transgenic mice expressing a sialic acid-specific 9-O-acetylase. Cell, 65: 65-74.

42. Birkle S, Gao L, Zeng G \& Yu RK (2000). Down regulation of GD3 ganglioside and its $\mathrm{O}$-acetylated derivative by stable transfection with antisense vector against GD3-synthase gene expression in hamster melanoma cells: effects on cellular growth, melanogenesis, and dendricity. Journal of Neurochemistry, 74: 547-554.

43. Walsh CA \& Goffinet AM (2000). Potential mechanisms of mutations that affect neuronal migration in man and mouse. Current 
Opinion in Genetics and Development, 10: 270-274.

44. Mendez-Otero R \& Constantine-Paton M (1990). Granule cell induction of 9-O-acetyl gangliosides on cerebellar glia in microcultures. Developmental Biology, 138: 400-409.

45. Hedin-Pereira C, deMoraes ECP, Lent R \& Mendez-Otero R (1997). Immunoblocking 9-O-acetylated gangliosides arrests cell migration in the developing cerebral cortex. Society for Neuroscience Abstracts, 23: 871 .

46. Komuro H, Yacubova E, Yacubova E \& Rakic P (2001). Mode and tempo of tangential cell migration in the cerebellar external granular layer. Journal of Neuroscience, 21: 527-540.

47. Ono K \& Kawamura K (1989). Migration of immature neurons along tangentially oriented fibers in the subpial part of the fetal mouse medulla oblongata. Experimental Brain Research, 78: 190-201.

48. Hu H, Tomasiewics H, Magnuson T \& Rutishauser U (1996). The role of polysialic acid in migration of olfactory bulb interneuron precursors in the subventricular zone. Neuron, 16: 735-743.

49. Mendez-Otero R \& Cavalcante LA (1996). Expression of 9-O-acetylated gangliosides is correlated with tangential cell migration. Neuroscience Letters, 204: 97-100.

50. Miyakoshi LM, Mendez-Otero R \& Hedin-Pereira C (2001). The 9-Oacetyl GD3 ganglioside is expressed by migrating chains of subventricular zone neurons in vitro. Brazilian Journal of Medical and Biological Research, 34: 669-673.

51. Miyakoshi LM, Mendez-Otero R \& Hedin-Pereira C (2001). Role of 9O-acetyl GD3 in the migration of subventricular zone neuronal precursors. Journal of Neurochemistry, 78 (Suppl): 56 (Abstract).

52. Gallo G \& Letourneau PC (1999). Axon guidance: a balance of signals sets axons on the right track. Current Biology, 9: R490R492.
53. Garcia-Abreu J, Mendes FA, Onofre GR, Freitas MS, Silva LCF, Moura Neto V \& Cavalcante LA (2000). Contribution of heparan sulfate to the non-permissive role of the midline glia to the growth of midbrain neurites. Glia, 29: 260-272.

54. Ferreira A, Busciglio J, Landa C \& Caceres A (1990). Gangliosideenhanced neurite growth: evidence for a selective induction of high molecular weight MAP-2. Journal of Neuroscience, 10: 293-302.

55. Mendez-Otero R \& Friedman JE (1996). Role of acetylated gangliosides on neurite extension. European Journal of Cell Biology, 71: 192-198.

56. Araujo H, Menezes MA \& Mendez-Otero R (1997). Blockage of 9-Oacetyl gangliosides induces depolymerization in growth cones and neurites. European Journal of Cell Biology, 72: 202-213.

57. Cheresh DA, Pierschbacher MD, Herzig MA \& Mujoo K (1986). Disialogangliosides GD2 and GD3 are involved in the attachment of human melanoma and neuroblastoma cells to extracellular matrix proteins. Journal of Cell Biology, 102: 688-696.

58. Probstmeier R \& Pesheva P (1999). Tenascin-C inhibits beta1 integrin-dependent cell adhesion and neurite outgrowth on fibronectin by a disialoganglioside-mediated signaling mechanism. Glycobiology, 9: 101-114.

59. Katz B-Z, Zamir E, Bershadsky A, Yamada KM \& Geiger B (2000). Physical state of the extracellular matrix regulates the structure and composition of cell-matrix adhesions. Molecular Biology of the Cell, 11: $1047-1060$

60. Kopitz J, Reitzenstein C, Burchert M, Cantz M \& Gabius HJ (1998). Galectin-1 is a major receptor for ganglioside GM1, a product of the growth-controlling activity of a cell surface ganglioside sialidase, on human neuroblastoma cells in culture. Journal of Biological Chemistry, 273: 11205-11211. 